\title{
Novel ETV1 mutation in small cell lung cancer transformation resistant to EGFR tyrosine kinase inhibitors
}

\author{
Yan Zhou ${ }^{1 \#}$, Hao Bai ${ }^{1 \#}$, Jinjing Xia ${ }^{1 \#}$, Wang-Yang $\mathrm{Xu}^{2}$, Lei Cheng ${ }^{1}$, Liwen Xiong ${ }^{1}$ \\ ${ }^{1}$ Pulmonary and Critical Care Medicine Department, Shanghai Chest Hospital, Shanghai Jiao Tong University, Shanghai, China; ${ }^{2}$ Medical \\ Department, Singlera Genomics (Shanghai) Ltd., Shanghai, China \\ Contributions: (I) Conception and design: L Xiong, Y Zhou; (II) Administrative support: L Xiong; (III) Provision of study materials or patients: H Bai, \\ J Xia; (IV) Collection and assembly of data: WY Xu; (V) Data analysis and interpretation: L Cheng; (VI) Manuscript writing: All authors; (VII) Final \\ approval of manuscript: All authors. \\ \#These authors contributed equally to this work. \\ Correspondence to: Liwen Xiong. Shanghai Chest Hospital, Shanghai Jiao Tong University, 241 West Huaihai Road, Shanghai 200030, China. \\ Email: Xiong_li_wen@126.com.
}

Background: Non-small cell lung cancer (NSCLC) patients harboring mutations in the epidermal growth factor receptor $(E G F R)$ gene respond dramatically to EGFR tyrosine kinase inhibitors (TKIs). However, these patients inevitably develop acquired resistance to EGFR-TKIs. Among them, small cell lung cancer (SCLC) transformation is a relatively rare mechanism.

Methods: We used a 639 cancer-relevant gene panel to detect genetic differences in tissues before and after EGFR-TKIs resistance caused by SCLC transformation. In vitro experiments were conducted to study the role of ETS variant transcription factor 1 (ETV1) on SCLC transformation and EGFR-TKIs resistance.

Results: We present two EGFR-mutant lung adenocarcinoma (LUAD) patients. One patient, with EGFR exon 19 deletion (Ex19del), accepted first-line gefitinib treatment and then received osimertinib treatment due to acquisition of an EGFR-T790M mutation. A novel ETV1 mutation (p.P159S) was detected in the SCLC tissue after osimertinib resistance when not coexisting with T790M. The other patient harbored an EGFR exon 21 mutation (p.L858R), and had a long-lasting response to first-line gefitinib, and then transformed to SCLC after TKI resistance. A previously unreported ETV1 mutation (p.E462Q) was detected in the SCLC tissue. In vitro, ETV1 p.E462Q and p.P159S mutations participated in neuroendocrine differentiation by inducing the expression of achaete-scute homolog 1 (ASCL1) and promoting the proliferation of H69 cells. ETV1 p.E462Q and p.P159S mutations were also resistant to gefitinib and osimertinib after introduction into $\mathrm{H} 358$ cells.

Conclusions: Novel ETV1 p.E462Q and p.P159S mutations were found in the SCLC tissues of TKIsresistant LUAD patients, providing a new understanding of ETV1 involvement in acquired resistance to EGFR-TKIs via SCLC transformation.

Keywords: ETV1; non-small cell lung cancer (NSCLC); small cell lung cancer transformation (SCLC transformation); EGFR tyrosine kinase inhibitor (EGFR TKI); drug resistance

Submitted Apr 27, 2021. Accepted for publication Jul 07, 2021.

doi: 10.21037/atm-21-2625

View this article at: https://dx.doi.org/10.21037/atm-21-2625

\section{Introduction}

Non-small cell lung cancer (NSCLC), which accounts for the majority of lung cancer cases, is one of the most common malignant tumors in the world $(1,2)$. Most of the patients are in advanced stage at the time of diagnosis, and those that harbor sensitive mutations could receive targeted therapies as the standard treatment. The most common epidermal growth factor receptor (EGFR) mutations are 
exon 19 (Ex19del) or exon 21 (L858R) (3). The use of first-line $E G F R$-tyrosine kinase inhibitors (TKIs) such as gefitinib, erlotinib (first-generation), and afatinib (secondgeneration), which represent the standard treatment of advanced EGFR-mutated NSCLC, dramatically improves the prognosis of patients (4). However, after a median of $8-12$ months of treatment, the disease will often progress, which indicates acquired resistance to EGFR-TKIs (5). The mechanisms of acquired drug resistance comprise EGFR T790M mutation, $c$-Met and HER2 amplification, and other driver gene mutations, as well as histological transformation to small cell lung cancer (SCLC) and an epithelial-mesenchymal (EMT) phenotype (6). Osimertinib (AZD9291) is the third generation of EGFR-TKIs, which has been shown to be effective in NSCLC patients with T790M mutation, however most patients will also inevitably develop drug resistance $(7,8)$. Acquired EGFR C797S mutation is one of the most common osimertinib-resistant mechanisms, and SCLC transformation has also been reported (9-11).

Studies have shown that TKIs-resistant SCLC basically retains the original EGFR mutations, supporting the view that SCLC is not an independent second primary cancer, while EGFR mutations after SCLC transformation are not sensitive to EGFR-TKIs (12). Moreover, the majority of patients with histologically transformed SCLC were female non-smokers, which differed from the clinical manifestations of primary SCLC patients. These studies hypothesized that SCLC and NSCLC may have common origin cells despite their different biological and genomic characteristics (12-14). SCLC transformation is always associated with inactivating mutations in the $R B 1$ and $T P 53$ genes (15). Other alterations such as PTEN, CREBBP, SLIT2, EP300, and MLL mutations, as well as FGFR1 amplification have also been found in SCLC transformation cases. Although these distinctive mechanisms are wellknown, a heterogeneity of the resistance to TKIs in individual is still under study and requires more attention.

Herein, we investigated two patients with EGFRmutant lung adenocarcinoma (LUAD) who had a longlasting response to gefitinib or osimertinib treatment and developed resistance to EGFR-TKIs due to SCLC transformation. We used a customized 639 cancer-related genes panel to analyze the genetic background of the tumor samples before EGFR-TKI treatment and after SCLC transformation, and detected novel ETV1 mutations in SCLC tissues. In vitro experiments showed that ETV1 mutations regulated achaete-scute homolog 1 (ASCL1) levels and conferred resistance to gefitinib and osimertinib, providing new insights into the role of ETV1 in lung tumorigenesis and histological transformation. This is the first report of a patient whose lung cancer transformed to SCLC after EGFR-TKI therapy with an acquired ETV1 mutations, which provides new insight into the function of the ETV1 gene and identifies a potential target that can be included in future treatment strategies for this type of cancer.

We present the following article in accordance with the MDAR reporting checklist (available at https://dx.doi. org/10.21037/atm-21-2625).

\section{Methods}

\section{Patients and sample collection}

Two NSCLC patients with EGFR sensitive mutations were recruited from the Shanghai Chest Hospital. A realworld study was conducted to detect genetic changes in the SCLC transformation after EGFR-TKI treatment. All procedures performed in this study involving human participants were in accordance with the Declaration of Helsinki (as revised in 2013). The study was approved by Research Ethics Committee of Shanghai Chest Hospital (IS2118) and informed consent was taken from all the patients. Clinical information including age at diagnosis, gender, tumor histology, clinical treatment approach, outcomes, and smoking status was collected. Formalin-fixed paraffin-embedded (FFPE) tumor tissues or fresh biopsy tissues were obtained. Histological types and tumor cell contents were confirmed by two pulmonary pathologists. The efficacy assessment which is divided into complete response (CR), partial response (PR), stable disease (SD) and progressive disease (PD) was evaluated using dedicated computed tomography imaging performed and evaluated by investigator according to the Response Evaluation Criteria in Solid Tumors (RECIST 1.1).

\section{Targeted next-generation sequencing (NGS) and bioinformatics analysis}

Germline DNA (gDNA) from fresh tissues or FFPE samples de-paraffinized with xylene were extracted by using the QIAamp DNA FFPE Tissue Kit (Qiagen, Hilden, Germany) according to the manufacturer's instructions. Sequencing libraries were prepared using the Illumina standard library (Illumina, Inc., California, USA) according to the 
manufacturer's protocols. Captured libraries were quantified by using the KAPA SYBR ${ }^{\circledR}$ FAST universal qPCR Kits (KAPA Biosystems, Boston, USA). The libraries were pairedend sequenced on an Illumina Miseq sequencer (Illumina, Hayward, CA, USA). A panel containing 639 cancer-related mutational hot genes (Singlera Genomics Inc., Shanghai, China) was used to detect multiple types of genomic variants, such as single-nucleotide variants, insertions, deletions, copy number variations, and rearrangements. The 639 involved genes are listed in Table S1.

Sequencing data were uploaded for filtering high quality reads, and clean data were aligned to The University of California at Santa Cruz (UCSC) human reference genome (GRCh37/hg19). Single-nucleotide variants, insertions or deletions, and amino acid changes were annotated by using the SnpEff (http://snpeff.sourceforge.net/). Variations detected in tumor tissues but not detected in matched blood were deemed as somatic alterations. Mutations with allele frequency $>5 \%$ were defined as novel mutations. The I-TASSER server (http://zhanglab.ccmb.med.umich.edu/ I-TASSER) was used to model the structure of ETV1.

\section{Cells and reagents}

The NCL-H358 cells (RRID: CVCL_1559) were maintained in RPMI1640 medium (Gibco BRL, Grand Island, NY, USA) supplemented with $10 \%$ fetal bovine serum (FBS, Gibco BRL, Grand Island, NY, USA) and $10 \mathrm{ng} / \mathrm{mL}$ of mouse IL3 (Cell Signaling Technology). NCL-H69 cells (RRID: CVCL_1579) were maintained in RPMI1640 medium (Gibco BRL, Grand Island, NY, USA) supplemented with $10 \%$ FBS (Gibco BRL). All cells were cultured at $37{ }^{\circ} \mathrm{C}$ in a $5 \% \mathrm{CO}_{2}$-humidified atmosphere. Gefitinib and Osimertinib were purchased from Selleck Chemicals (Houston, TX, USA).

\section{Plasmid construction}

A full-length cDNA fragment of human EGFR containing activating mutations (p.L858R and p.T790M) and a fulllength cDNA fragment of human ETV1 containing activating mutations (p.P159S and p.E462Q) were generated by Asia-Vector Biotechnology (Shanghai) Co. LTD. All mutated full-length EGFR or ETV1 cDNAs were induced into the PCDNA3.1 vector (Asia-Vector Biotechnology, Shanghai, China) and confirmed by direct sequencing. The sequences for mutagenesis primers are listed in Table S2.

\section{Cell growth inbibition assay}

A total of $10^{4}$ transfected $\mathrm{H} 358$ cells expressing different EGFR-mutant variants were plated in each well of a 96-well plate, which were grown in RPMI1640 medium. Next, dimethyl sulfoxide (DMSO) or EGFR-TKIs were added at the indicated drug doses, and the cells were cultured for 72 hours. The inhibitory effects of gefitinib and osimertinib on cell growth were detected using the Cell Counting Kit-8 reagent (BBI Life Sciences, Shanghai, China). Each experiment was performed in triplicate.

\section{Proliferation assay}

A total of $10^{4}$ cells were plated in each well of a 96-well plate and grown in RPMI1640 medium with 10\% FBS. At the indicated times points, the cell proliferation rate was detected using the Cell Counting Kit- 8 reagent (BBI Life Sciences, Shanghai, China) according to the manufacturer's protocol. The number of viable cells was counted, and the proliferation plot was drawn using GraphPad Prism 8.0 software (GraphPad Software Inc. San Diego, CA, USA). Each experiment was performed in triplicate.

\section{Quantitative reverse transcription PCR (qRT-PCR)}

Total ribonucleic acid (RNA) was extracted using Trizol reagent (Invitrogen, CA, USA), and was then reverse transcribed into cDNA using the reverse transcriptase reagent kit (TaKaRa, Shiga, Japan). Quantitative PCR was performed using SYBR green PCR kit (TaKaRa, Shiga, Japan). Relative transcript quantities were analyzed using the $\Delta \Delta$ cycle threshold ( $\triangle \Delta \mathrm{CT}$ ) method and CT values were normalized to the CT value of the GAPDH gene. Data from each experiment was then normalized to the wild-type (WT) group to detect the relative expression changes in messenger RNA (mRNA). Each experiment was performed in triplicate. The sequences of primers are listed in Table S2.

\section{Statistical analysis}

For in vitro experiments, quantitative data were presented as mean \pm standard errors of the mean. A two-tailed Student $t$-test was performed to calculate the statistically significant differences between two groups. A P value $<0.05$ was considered statistically significant. For all figures: asterisk $\left(^{*}\right)$ 
Table 1 Clinical characteristics of the two patients

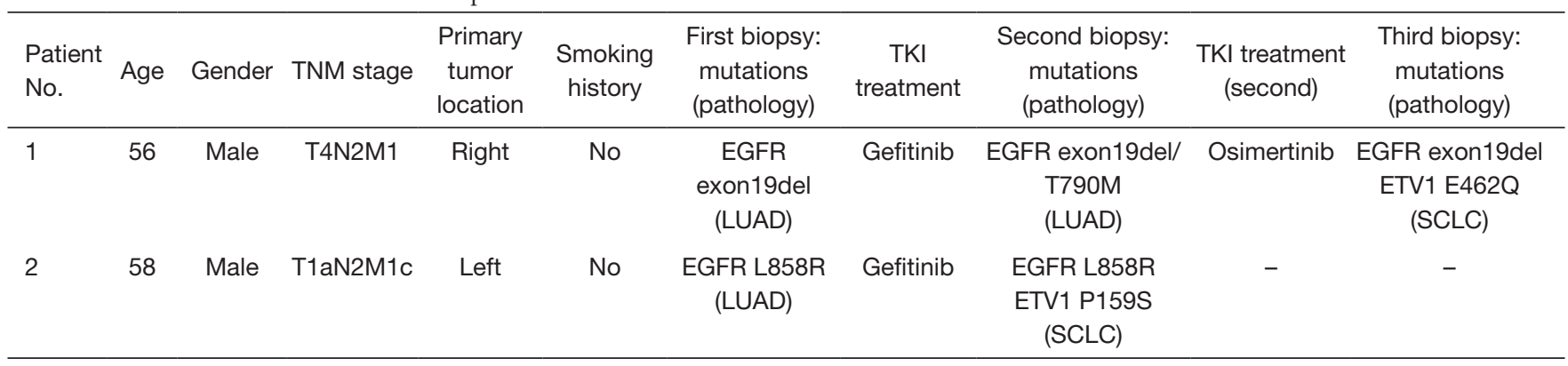

means $\mathrm{P}$ value $<0.05,\left(^{* *}\right)$ means $\mathrm{P}$ value $<0.01,\left({ }^{* * *}\right)$ means $\mathrm{P}$ value $<0.001$.

\section{Results}

\section{Patient's description}

We report that two patients diagnosed with LUAD carrying EGFR activating mutations received EGFR-TKIs. Nonetheless, the patients progressed and tissue re-biopsies immediately following progression revealed a switch to SCLC histology. The clinical characteristics of the two individuals are shown in Table 1.

\section{Case 1}

A 56-year-old male, who never smoked, had intermittent hemoptysis for 5 months. Computed tomography (CT) showed an enlarged pleural mass in the inferior lobe of the right lung $(4.3 \mathrm{~cm} \times 2.9 \mathrm{~cm})$ and multiple small nodules in bilateral lungs (Figure 1A). The pathological diagnosis was LUAD stage IV (T4N2M1), and immunohistochemical staining (IHC) confirmed the histology of pulmonary LUAD [pan cytokeratin positive $(\mathrm{CK})+$, thyroid transcription factor-1 positive (TTF1)+, cluster of differentiation 56 (CD56)-, and $\mathrm{Ki}-67$ 60\%]. The molecular pathology results showed EGFR exon 19 deletion (2235_2249del/E746_A750del). This patient began firstline treatment with gefitinib $250 \mathrm{mg}$, once a day orally. CT scans showed a response after three months of treatment, and gefitinib was continued for a total of 12.5 months until the patient developed right pleural effusion (Figure $1 \mathrm{~A}$ ).

Subsequently, an EGFR T790M mutation was detected via the 639 DNA panel, and IHC showed CD56-, CK+, TTF1+, and NapsinA+ in the second biopsy specimen (Figure S1A). Osimertinib $80 \mathrm{mg}$, once daily orally, was then administered. After 4 months, osimertinib was permanently discontinued because of multiple localized pleural thickening on the right side of the lung (Figure $1 A$ ). A re-biopsy (third biopsy) was then performed, and this tumor was diagnosed as SCLC with a pathological stage of IV (T4N2M1) according to the World Health Organization (WHO) 2015 classification of lung tumors. IHC showed CD56+, CK+/-, TTF1+ (partial), NapsinA-, and synaptophysin+ (Figure S1B). Molecular analysis revealed novel genetic alterations different from those detected in previous biopsies, such as the ETV1 p.E462Q mutation. The patient then received standard carboplatin $(350 \mathrm{mg}$ ) and etoposide $(100 \mathrm{mg} /$ day $\times 5$ days $)$ therapy. After four cycles of this combined treatment, the patient exhibited disease progression and gradual increase of pleural effusion (Figure 1A).

\section{Case 2}

A 58-year-old man with no smoking history, complained of cough, sputum, and bone pain at his primary hospital. CT showed a $1.6 \mathrm{~cm}$ mass in the upper lobe of the left lung, bilateral pleural thickening, and uneven bone mineral density of the left scapula and part of the vertebral body (Figure 1B). Positron emission tomography-CT (PET-CT) revealed multiple lymph node metastases in the mediastinum, left hilum, and armpit, as well as bone metastasis. A CT-guided biopsy from the primary lesion was performed and diagnosed as LUAD with a pathological stage of IV (T1aN2M1c). IHC showed CD56-, CK+, and TTF1+ (Figure S1C). EGFR L858R was positively detected by NGS, and gefitinib $250 \mathrm{mg}$, once daily orally, was administered as the first-line therapy, resulting in a partial response $(\mathrm{PR})$ (Figure $1 B$ ).

After 64 months, the left pleural dissemination began to grow (Figure 1B). After hospitalization, re-biopsy was performed from the pleural tumor near the left diaphragm, and transformation to SCLC with a pathological stage of IV (T2aN2M1c) was confirmed. IHC showed CD56+, CK+, TTF1+, and NapsinA+ (Figure S1D). T790M was not 

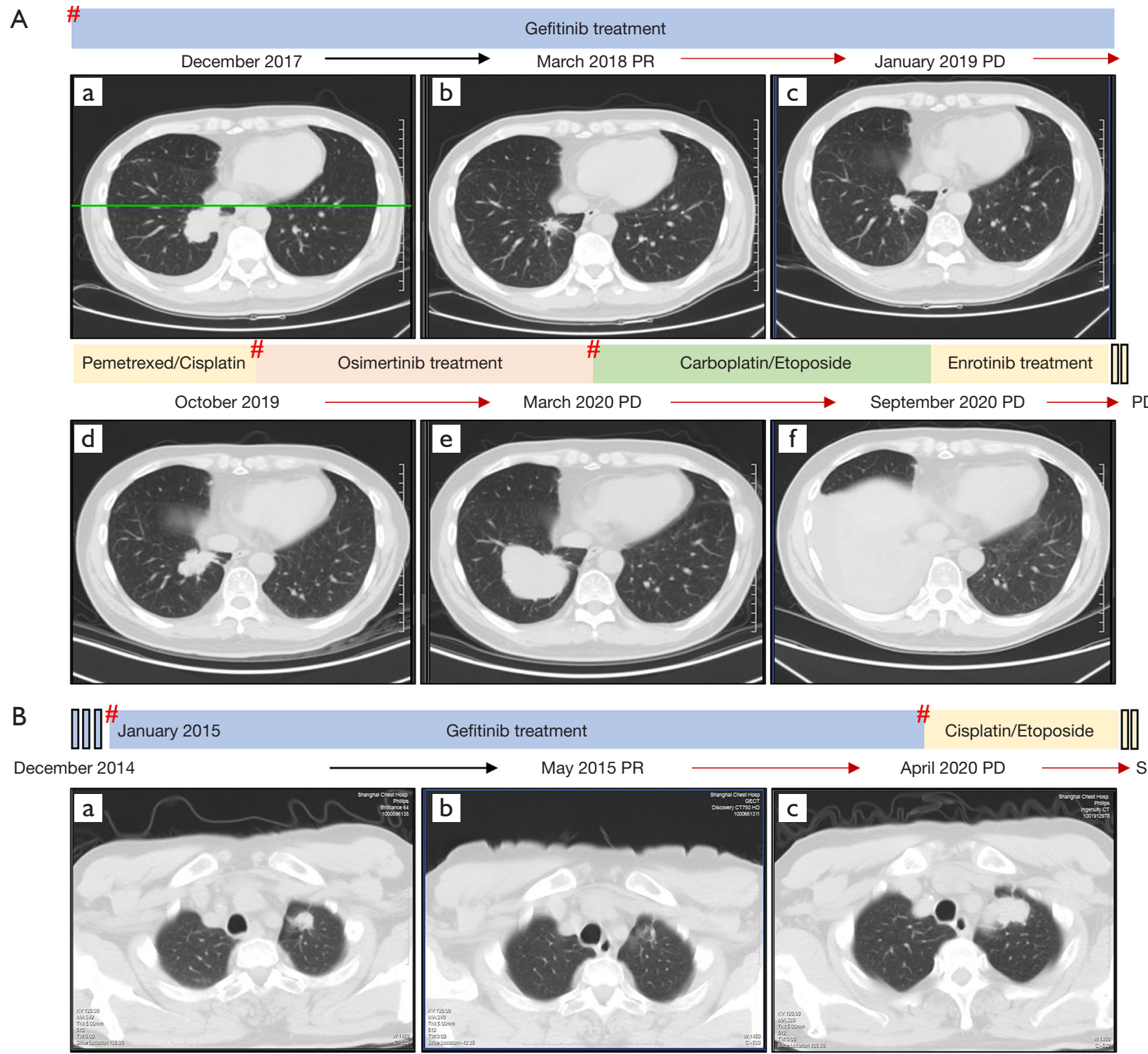

Gefitinib treatment

\# Cisplatin/Etoposide

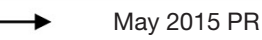

April 2020 PD
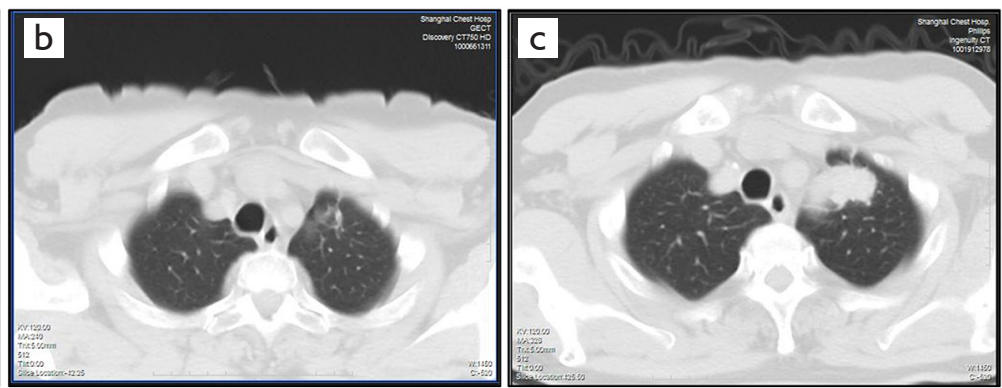

Figure 1 Tumor lesions detected by CT during the courses of the patients' diseases and treatment. (A) Case 1: (a) Before start of EGFR-TKI treatment. NGS showed EGFR exon 19 deletion. (b) PR after gefitinib treatment. (c) First progression after 12.5 months of gefitinib. (d) PD after 9 months of chemotherapy. EGFR T790M was detected. (e) Second progression after 4 months of osimertinib treatment. Appearance of the transformation to SCLC. ETV1 p.E462Q was detected. (f) PD after 6 months of chemotherapy. (B) Case 2: (a) Before start of EGFRTKI treatment. EGFR L858R was detected. (b) PR after 4 months of gefitinib treatment. (c) PD after 64 months of gefitinib treatment. ETV1 p.P159S was detected. " represents the time point of NGS detection. NGS, next-generation sequencing; SCLC, small cell lung cancer; PR, partial response; PD, progressive disease.

detected in the tissue, however the novel ETV1 mutation (ETV1 p.P159S) was, as in the SCLC tissue analysis of patient 1 . Treatment was then changed to chemotherapy (cisplatin $100 \mathrm{mg}+$ etoposide $100 \mathrm{mg} /$ day $\times 5$ days), which has been used until now.

\section{Mutational analysis of ETV1}

ETV1, which is located in $7 \mathrm{p} 21.2$, contains 21 exons and encodes a member of the ETS (E twenty-six) family of transcription factors. ETS transcription factors can directly bind to specific DNA sequences in the promoter/enhancer 
regions of genes to regulate various biological processes (16). The Cancer Genome Atlas (TCGA) database showed that ETV1 mutations were most common in skin cancer, bladder urothelial cancer, nerve sheath tumor, prostate adenocarcinoma, melanoma, and endometrial carcinoma (Figure 2A). Point mutations reported in tumors were scattered in ETV1 (Figure 2B). The mutation frequency of ETV1 in LUAD and SCLC was $3.64 \%$ and $0.84 \%$, respectively (Figure 2C,D). The mutation frequency of ETV1 reported in the Asian population with LUAD was $1.37 \%$, while that of European and Latin American populations was $2.73 \%$ and $1.71 \%$, respectively. The point mutations of ETV1 of LUAD were mainly concentrated in the ESTPEA3-N domain (Figure 2E).

In TCGA database, only three SCLC patients were reported to have ETV1 mutation, and all of them were ETV1 S100G (Figure 2E). The ethnic origin of samples with ETV1 mutations in SCLC is unknown. Survival analysis showed that patients with ETV1 mutations had a worse overall survival (OS) trend than those without ETV1 mutations, although this was not statistically significant (Figure S2A). However, no differences were observed in progression-free survival (PFS) (Figure S2B). The worse OS trend of LUAD patients with ETV1 mutations was obvious (Figure S2C), and the disease-free survival (DFS) of these patients was significantly shortened compared to LUAD patients without ETV1 mutations (Figure S2D). SCLC patients had insufficient data to show the correlation between ETV1 mutations and survival.

Clinical correlation analysis indicated that ETV1 mutations were significantly associated with advanced Tumor Node Metastasis (TNM) stages of LUAD $(\mathrm{P}=0.038)$ (Figure S3A). ETV1 mutations were more common in never-smoking LUAD patients (Figure S3B). In this study, the novel ETV1 mutations identified in two cases were ETV1 Pro159Ser (P159S), which was located in ESTPEA3-N domain, and Glu462Gln (E462Q), which was not located in a common functional domain (Figure $3 A$ ). Both of them changed the structures of the protein by computer simulation (Figure $3 B, C$ ), illustrating an important role of ETV1 in the development of lung cancer that has not been previously reported.

\section{ETV1 mutations induced ASCL1 expression in SCLC}

To ascertain whether ETV1 participated in neuroendocrine differentiation through regulating the expression of ASCL1, we transfected ETV1 mutant plasmids into H69 cells. We found that there was no significant difference in the expression of ETV1 mRNA (Figure 4A), while ETV1 p.E462Q and p.P159S mutant plasmids increased the ASCL1 mRNA levels after transfection (Figure 4B). We discovered that ETV1 mutations indirectly inhibited HES1 transcription (Figure 4C).

\section{ETV1 mutations promoted SCLC proliferation in vitro}

We infected H69 cells with mutant ETV1 plasmids and found that ETV1 p.E462Q and p.P159S mutations promoted SCLC proliferation in vitro. On the $2^{\text {nd }}$ day after transfection, we observed that ETV1 p.E462Q and p.P159S mutations markedly induced the proliferation of tumor cells (Figure 4D).

\section{ETV1 p.E462Q and p.P159S mutations conferred resistance to gefitinib and osimertinib in vitro}

We next determined whether ETV1 p.E462Q and p.P159S mutations contributed to EGFR-TKI resistance. We transfected plasmids harboring EGFR L858R or T790M (in cis) with or without ETV1 p.E462Q or p.P159S mutations into H358 cells, and exposed these cells to increased doses of osimertinib or gefitinib. As expected, cells expressing the EGFR L858R mutation were sensitive to gefitinib and osimertinib, with an IC50 of 241.8 and $160.3 \mathrm{nM}$, respectively (Figure 5A,B). Cells containing EGFR T790M were sensitive to osimertinib. EGFR mutant cells transfected with the ETV1 p.P159S mutant variant exhibited a strong resistance to osimertinib. In cells exposed to increased doses of gefitinib, the ETV1 p.E462Q and p.E462Q mutant variants exhibited a strong resistance to gefitinib when EGFR T790M was negative (Figure 5C).

\section{Discussion}

A large number of advanced NSCLC patients with EGFR mutations exhibit a good response to EGFR-TKIs. However, most patients develop resistance after an average of $8-14$ months, caused by various alterations including EGFR T790M mutation, EGFR amplification, MET and HER2 amplification, and PIK3CA mutations (13). Furthermore, $5-15 \%$ of NSCLC tumors will transform into SCLC histology $(13,17)$. Acquired resistance is the main problem that limits the clinical effect of targeted treatment with EGFR-TKIs. Although the response of transformed SCLC to chemotherapy is good, the prognosis 


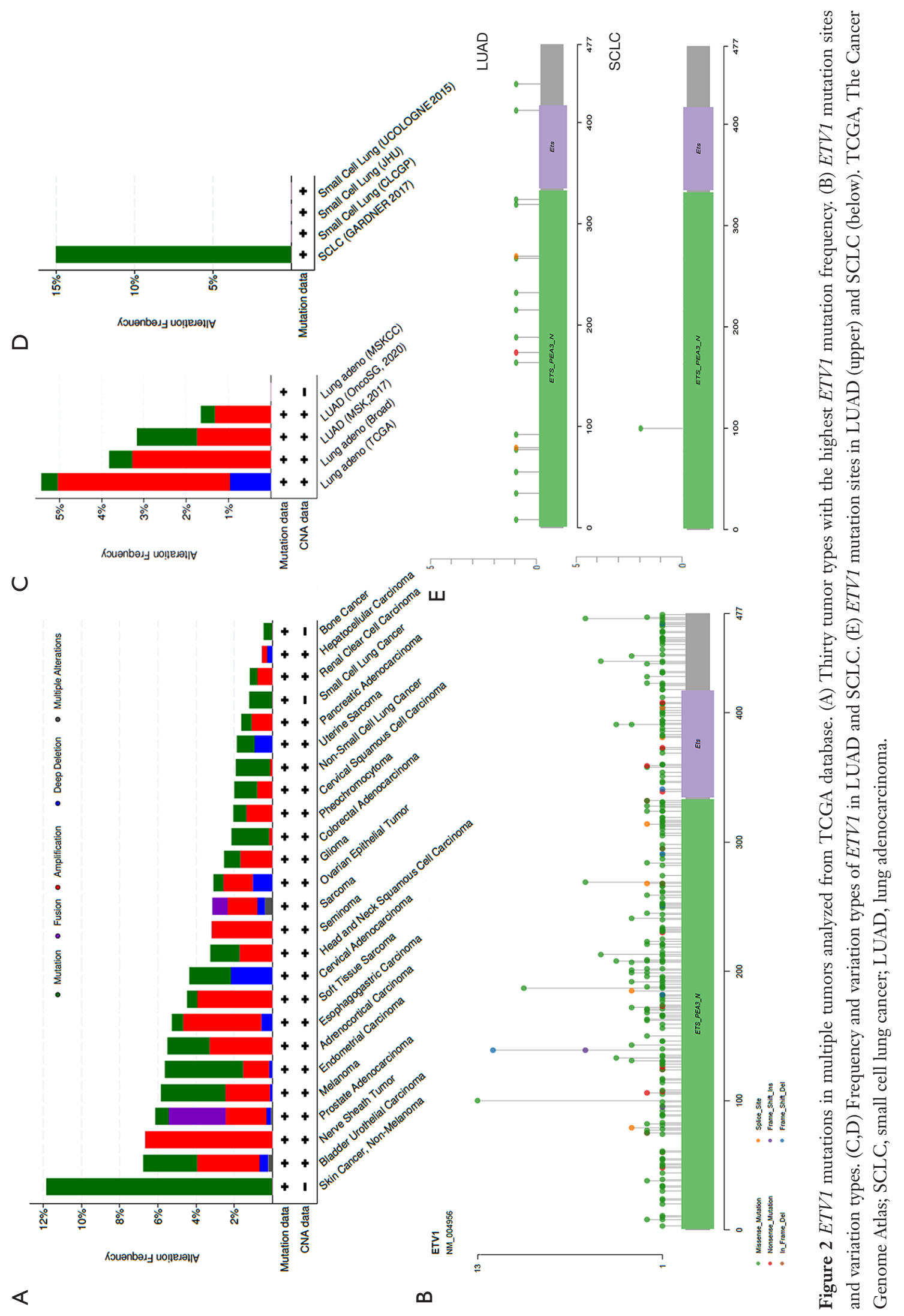



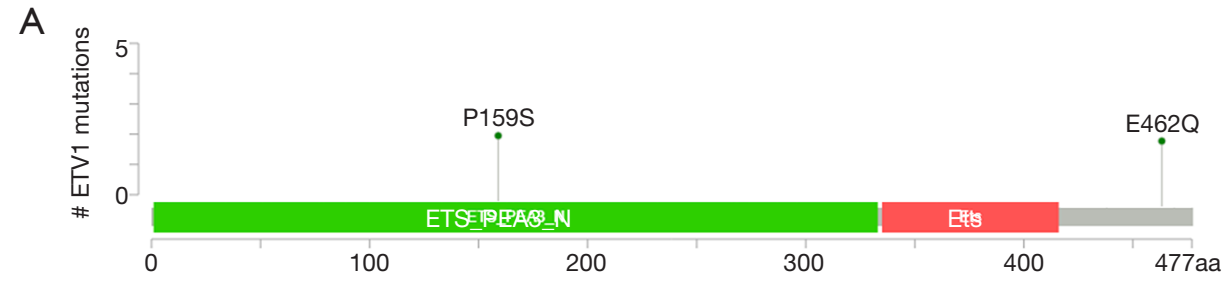

B
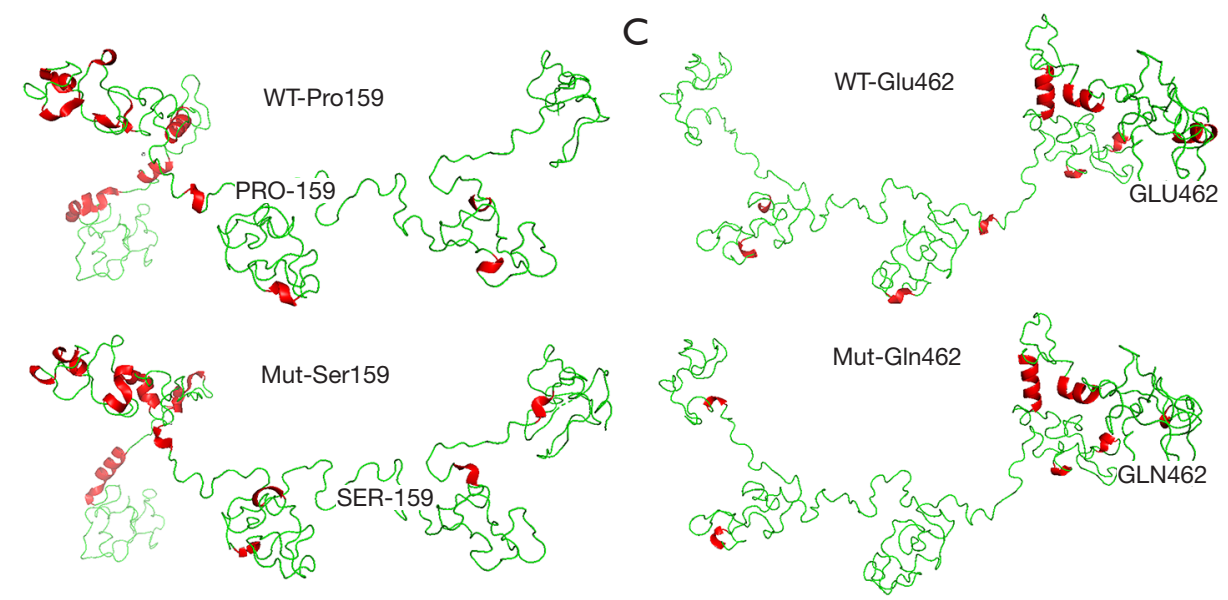

Figure 3 Structure domain and 3D protein structure diagrams of ETV1. (A) Two mutant sites were indicated in the structure of ETV1. (B,C) 3D protein structures of normal and mutant proteins of ETV1. Red means helix, green means sheet. ETV1, ETS variant transcription factor 1 .
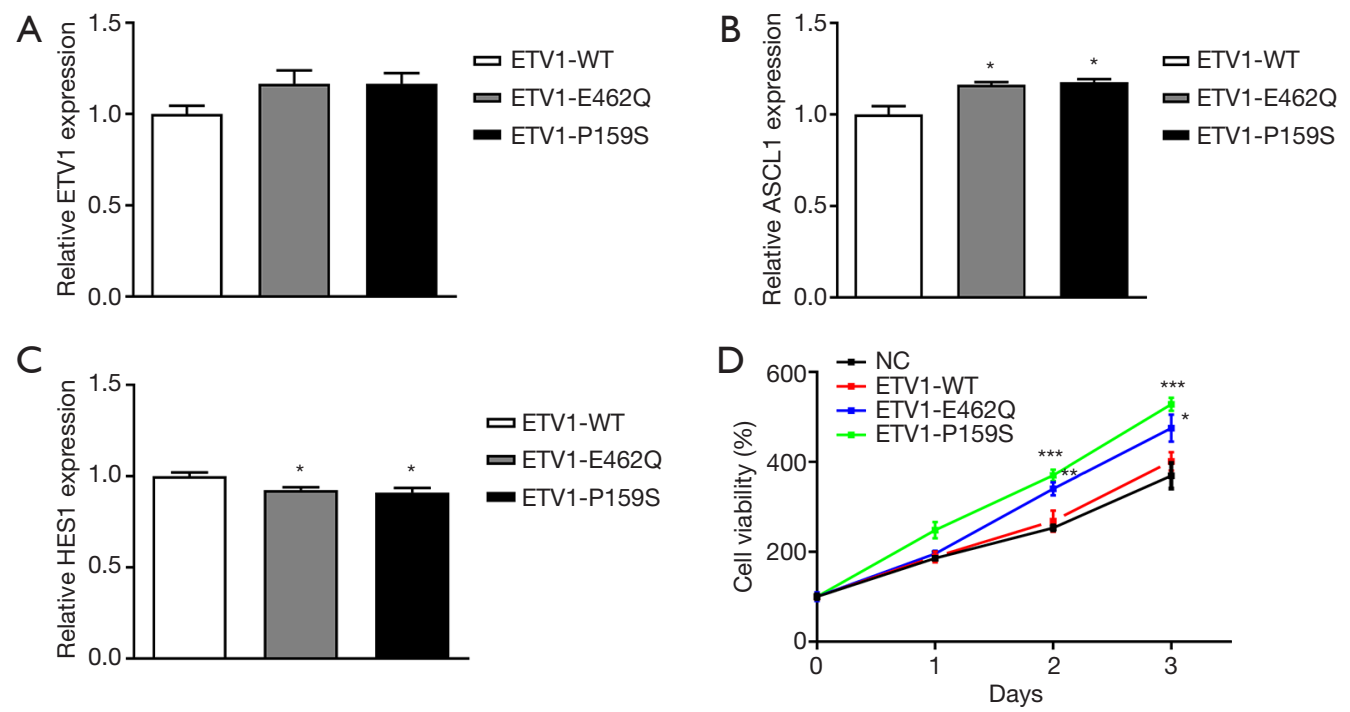

Figure 4 ETV1 p.E462Q and p.P159S mutations promoted ASCL1 expression in SCLC. (A) Real time-PCR was used for the mRNA expression of ETV1 after transfection of the ETV1 WT and mutant plasmids. (B,C) ASCL1 and HES1 mRNA levels in cells transfected with ETV1 mutant plasmids compared with those in cells transfected with the ETV1 WT plasmid. (D) Cell proliferation of H69 cells after transfection of the ETV1 WT and mutant plasmids. * $\mathrm{P}<0.05$; **, $\mathrm{P}<0.01$; ***, $\mathrm{P}<0.001$. ASCL1, achaete-scute homolog 1; SCLC, small cell lung cancer; ETV1, ETS variant transcription factor 1; WT, wild-type. 
A

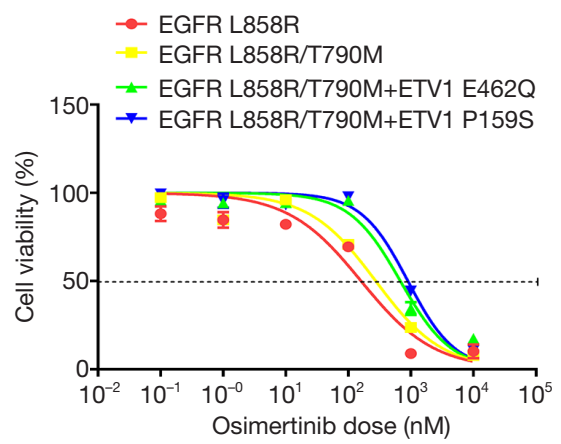

C

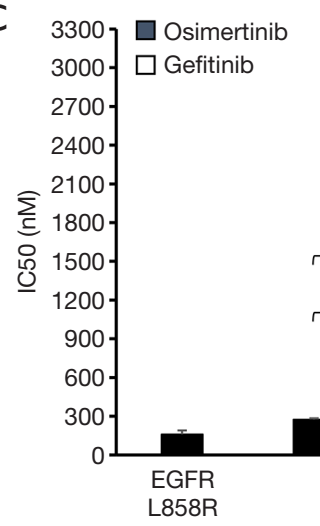

B

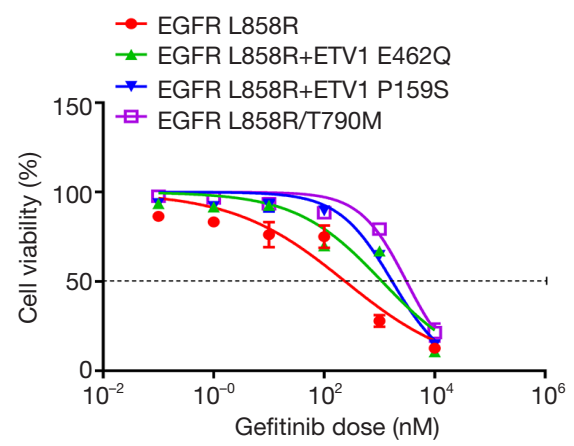

\EGFR L858R+ETV1 E462Q

$\rightarrow$ EGFR L858R+ETV1 P159S

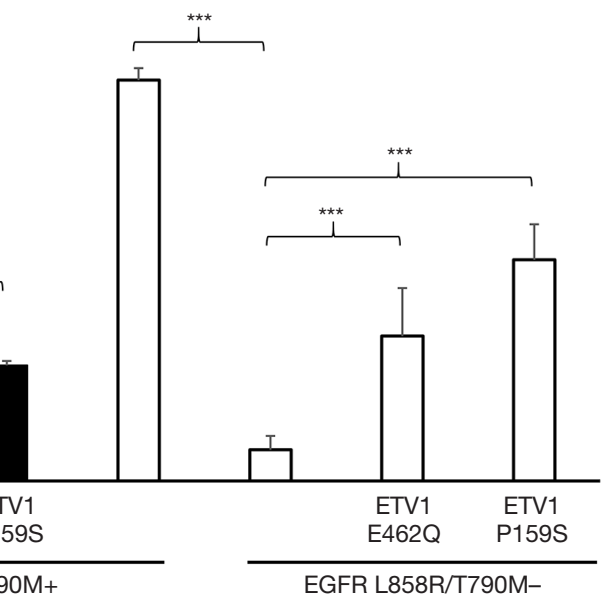

EGFR L858R/T790M+

EGFR L858R/T790M-

Figure 5 ETV1 p.E462Q and p.P159S mutations induced gefitinib and osimertinib resistance in vitro. H358 cells harboring EGFR L858R and T790M plus indicated mutations were treated with osimertinib (A) or gefitinib (B) at the indicated concentrations. Cell viability was detected after 24 hours of treatment and plotted for comparison with untreated control cells. (C) IC50 values of different EGFR mutant cell lines to osimertinib and gefitinib treatment were plotted in bar graphs for comparison. Experiments were repeated twice in triplicate each time and the mean \pm standard deviation value was plotted at each concentration. ${ }^{* * *}, \mathrm{P}<0.001$. EGFR, epidermal growth factor receptor; ETV1, ETS variant transcription factor 1.

is poor $(18,19)$. The median survival time after confirmed transformation is 6-7 months (18). From a historical point of view, it is surprising that tumors could differentiate into NSCLC or SCLC at an early stage of tumorigenesis. EGFR mutant adenocarcinoma mainly occurs in people who never smoke, accompanied by a more sluggish natural process. Compared with NSCLC, SCLC almost always occurs in heavy smokers, and is prone to early metastasis and rapid growth $(13,20)$.

In this study, repeated biopsies reminded us that SCLC transformed tumors might evolve from the initial adenocarcinoma rather than a concurrent cancer in the initial cases, because $E G F R$ mutations in all cases were the same as those in the original LUAD. However, we could not rule out the possibility that SCLC with EGFR mutation existed before EGFR-TKI treatment, although we did not observe a mixed histology of NSCLC and SCLC in the tumor before treatment after carefully reviewing the histology of these two samples. However, from a clinical point of view, we suggested that these SCLCs were unlikely to exist in the early stage of tumorigenesis because classical SCLC develops very rapidly (13). RB expression loss and TP53 mutation were deemed as the main molecular mechanisms involved in predicting SCLC transformation (21). After TKIs treatment, gene alterations accumulated, and EGFR signal that stimulated NSCLC differentiation was no longer necessary for proliferation, so cells differentiated into other lineages, including EGFR mutant SCLC. In addition, alveolar type II cells can produce EGFR mutant adenocarcinoma, but after TP53 and $R B 1$ interfere with alveolar type II cells, SCLC appears. Other alterations such as PTEN, CREBBP, SLIT2, EP300, 
and $M L L$ mutations, as well as FGFR1 amplification have also been found in SCLC transformation cases $(22,23)$. In view of these changes after EGFR-TKI exposure, resistant pluripotent stem cells subsequently differentiate into SCLC cells. Since these cells do not need EGFR signaling, they escape the influence of EGFR-TKI, so they will proliferate despite the presence of EGFR-TKI. Although there may be some EGFR mutations in transformed SCLC, the decrease or absent expression of EGFR may also lead to poor response to EGFR-TKI. The analysis of EGFR mutant tumor from adenocarcinoma to SCLC as acquired resistance mechanism showed that the lose protein expression of EGFR and the amplification level of EGFR was low. So, the response of EGFR mutant SCLC to $E G F R$-TKI does not seem to match those of EGFR-mutant adenocarcinoma. Thus, it is generally not recommended to detect EGFR mutations in SCLC. TKIs may not be effective in patients with SCLC due to the loss of EGFR expression at the protein level unless rare case had a positive EGFR mutation status, and they may respond to EGFR TKIs. In these two cases, ETV1 alterations were particularly related to the transformation to SCLC, which provides new insight into the function of the ETV1 gene and identifies a potential target that can be included in future treatment strategies for this type of cancer.

ETV1 is known as an oncogenic driver, and manifests as genomic translocation or amplification that causes aberrant E-twenty-six or E26 transformation-specific (ETS) expression in Ewing sarcoma, prostate cancer, and gastrointestinal stromal tumors, but not in lung cancer. In addition, ETV1 fusion has been previously described in prostate cancer, melanoma, and Ewing sarcoma (24). Recently, PTPRZ1-ETV1 and DGKB-ETV1 fusion have also been detected in glioma $(24,25)$. Herein, we first presented two cases in which ETV1 mutations and neuroendocrine transformation exist together in the same lesion. Thus, to our knowledge, ETV1 p.E462Q and p.P159S mutations detected in these two cases have not previously been reported in lung or other tumors. The prediction of the PROVEAN software (http://provean.jcvi.org) indicated that the mutant protein had a high score of pathogenicity and was a "deleterious" mutation, so these attracted our attention.

According to the LUAD data, there was no significant difference in the mutation frequency of ETV1 between LUAD and SCLC, but there were fewer ETV1 mutant cases reported in SCLC and the mutation was single. The mutation frequency of ETV1 reported in the Asian population with LUAD was $1.37 \%$, while that of European and Latin American populations was $2.73 \%$ and $1.71 \%$, respectively. Only three SCLC patients with LUAD had the same ETV1 mutation. However, there was no case report of ETV1 mutations associated with SCLC type transition. All of these indicate that ETV1 mutations are rare in lung cancer, however the role of ETV1 gene in the occurrence and development of lung cancer is worth studying. In particular, computer simulation suggested that these two ETV1 mutations might cause structural changes to the ETV1 protein, which may affect the normal function of enzyme activation and DNA binding.

The maintenance of the neuroendocrine phenotype of SCLC could be detected by the presence of neuroendocrine markers, such as ASCL1. The expression of ASCL1, which was obviously present in the SCLC components while being absent or rarely present in the LUAD, highlighted the vital role of ASCL1 in small cell transformation (26). In this study, in vitro experimentation showed that ETV1 mutations increased the mRNA expression of ASCL1 in SCLC and promoted SCLC proliferation.

We also found that ETV1 mutations could confer remarkable resistance to gefitinib and osimertinib. The ETV1 p.E462Q variant exhibited mild resistance to osimertinib, while the ETV1 p.E462Q and p.P159S variants showed strong resistance to gefitinib. Previous research has demonstrated that oncogenic ETV1/4/5 are targets of Capicua (CIC), a transcription factor downstream of the receptor tyrosine kinase and mitogen-activated protein kinase pathways, which have been found to play an important role in CIC loss mediating resistance to EGFR inhibition (27). ETV1 expression is known to regulate ASCL1 expression via Notch signaling $(28,29)$. We detected the expression levels of HES1, and suggested that ETV1 mutations regulated the expression of ASCL1 through Notch, which may be proven in further study. Ultimately, there was no significant change in the ETV1 mRNA levels after transfection of the ETV1 mutant plasmids. Collectively, these observations suggest the possibility that ETV1 mutations may represent a mechanism of intrinsic resistance to EGFR-TKIs (Figure 6). Due to the heterogeneity of tumors, it is conceivable that multiple drug resistance mechanisms may exist simultaneously in a single patient, except for SCLC transformation.

The first-line standard chemotherapy is etoposide or irinotecan combined with platinum. Concurrent or sequential radiotherapy of the thorax and mediastinum is also necessary at a limited stage. If complete remission, 


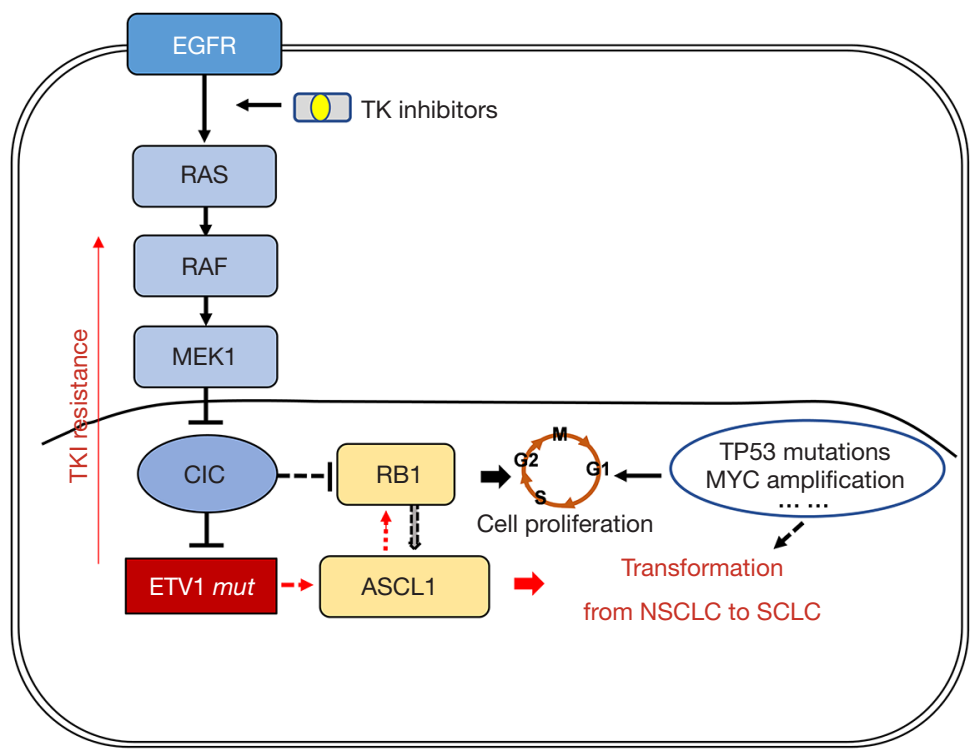

Figure 6 Molecular mechanisms of ETV1 resistance to EGFR tyrosine kinase inhibitors in SCLC transformation. ETV1 mutations stimulated EGFR signaling during gefitinib and osimertinib treatment and indirectly promoted ASCL1 overexpression, which contributed to neuroendocrine differentiation. EGFR, epidermal growth factor receptor; SCLC, small cell lung cancer; NSCLC, non-small cell lung cancer; ETV1, ETS variant transcription factor 1; ASCL1, achaete-scute homolog 1.

prophylactic cerebral irradiation (PCI) can prevent subsequent brain metastasis. In the extensive stage, chemotherapy is the main means of first-line treatment. SCLC is usually sensitive to initial treatment while most patients have recurrent disease and usually have additional metastases after initial treatment. Unfortunately, few drugs have been approved as effective second-line treatment for SCLC and the effects of them are not so good. In recent years, targeted therapy and immunotherapy have been actively tried. Nivolumab, the first FDA approved thirdline treatment for SCLC, pembrolizumab, atezolizumab and durvalumab have achieved encouraging results. For other treatment options, the cytotoxic drug lurbinectedin was granted orphan drug. In addition, epigenetic inhibitors such as EZH1/2 inhibitors bring hope for the treatment of SCLC. The prospect of better treatment of SCLC lies in the combination of immunotherapy and small molecule TKI drugs. However, the success of this strategy will require the use of validated biomarkers to select the patients most likely to benefit from this strategy, including NGS for molecular alterations and a second biopsy to guide the next step of treatment and predict the prognosis.

This study highlights the importance of re-biopsy of progressive tumors in identifying a heterogeneous histopathological and genetic mechanism of resistance that might occur in EGFR-mutated NSCLCs when a rapid progression exists after an initial response. This will help clinicians to formulate the next stage of therapy, as the treatment of advanced LUAD and SCLC is completely different. In this study, the transformed SCLCs exhibited a good response to initial etoposide and cisplatin (EP) chemotherapy. Other drugs for transformed SCLC or other transformed subtypes need to be comprehensively explored in the future.

\section{Conclusions}

In this study, novel ETV1 mutations were identified in two cases of EGFR-mutant LUAD that transformed to SCLC during treatment with first- and third-generation EGFR-TKIs. ETV1 contributed to the promotion of neuroendocrine differentiation by stimulating ASCL1 expression and cell proliferation in SCLC. Moreover, ETV1 could confer resistance to gefitinib and osimertinib in vitro. In future studies, the biological function of ETV1 in lung cancer requires further exploration. 


\section{Acknowledgments}

We are particularly grateful to the participants in this research.

Funding: This work was supported by the Scientific Research Project of Shanghai Municipal Health Commission (201940084), the Beijing Medical and Health Foundation (YWJKJJHKYJJ-F2213E), and the Shanghai Sailing Program (20YF1444600).

\section{Footnote}

Reporting Checklist: The authors have completed the MDAR reporting checklist. Available at https://dx.doi. org/10.21037/atm-21-2625

Data Sharing Statement: Available at https://dx.doi. org/10.21037/atm-21-2625

Conflicts of Interest: All authors have completed the ICMJE uniform disclosure form (available at https://dx.doi. org/10.21037/atm-21-2625). WYX reports that she was employed by company Singlera Genomics (Shanghai) Ltd. The other authors have no conflicts of interest to declare.

Ethical Statement: The authors are accountable for all aspects of the work in ensuring that questions related to the accuracy or integrity of any part of the work are appropriately investigated and resolved. All procedures performed in this study involving human participants were in accordance with the Declaration of Helsinki (as revised in 2013). The study was approved by Research Ethics Committee of Shanghai Chest Hospital (IS2118) and informed consent was taken from all the patients.

Open Access Statement: This is an Open Access article distributed in accordance with the Creative Commons Attribution-NonCommercial-NoDerivs 4.0 International License (CC BY-NC-ND 4.0), which permits the noncommercial replication and distribution of the article with the strict proviso that no changes or edits are made and the original work is properly cited (including links to both the formal publication through the relevant DOI and the license). See: https://creativecommons.org/licenses/by-nc-nd/4.0/.

\section{References}

1. Jemal A, Bray F, Center MM, et al. Global cancer statistics.
CA Cancer J Clin 2011;61:69-90.

2. Bade BC, Dela Cruz CS. Lung Cancer 2020:

Epidemiology, Etiology, and Prevention. Clin Chest Med 2020;41:1-24.

3. Chen HF, Lei L, Wu LX, et al. Effect of icotinib on advanced lung adenocarcinoma patients with sensitive EGFR mutation detected in ctDNA by ddPCR. Transl Cancer Res 2019;8:2858-63.

4. Novello S, Barlesi F, Califano R, et al. Metastatic nonsmall-cell lung cancer: ESMO Clinical Practice Guidelines for diagnosis, treatment and follow-up. Ann Oncol 2016;27:v1-v27.

5. Jackman D, Pao W, Riely GJ, et al. Clinical definition of acquired resistance to epidermal growth factor receptor tyrosine kinase inhibitors in non-small-cell lung cancer. J Clin Oncol 2010;28:357-60.

6. Facchinetti F, Proto C, Minari R, et al. Mechanisms of Resistance to Target Therapies in Non-small Cell Lung Cancer. Handb Exp Pharmacol 2018;249:63-89.

7. Buder A, Hochmair MJ, Setinek U, et al. EGFR mutation tracking predicts survival in advanced EGFR-mutated nonsmall cell lung cancer patients treated with osimertinib. Transl Lung Cancer Res 2020;9:239-45.

8. Mok TS, Wu YL, Ahn MJ, et al. Osimertinib or PlatinumPemetrexed in EGFR T790M-Positive Lung Cancer. N Engl J Med 2017;376:629-40.

9. Bordi P, Del Re M, Minari R, et al. From the beginning to resistance: Study of plasma monitoring and resistance mechanisms in a cohort of patients treated with osimertinib for advanced T790M-positive NSCLC. Lung Cancer 2019;131:78-85.

10. $\mathrm{Mu} \mathrm{Y,} \mathrm{Hao} \mathrm{X,} \mathrm{Xing} \mathrm{P,} \mathrm{et} \mathrm{al.} \mathrm{Acquired} \mathrm{resistance} \mathrm{to}$ osimertinib in patients with non-small-cell lung cancer: mechanisms and clinical outcomes. J Cancer Res Clin Oncol 2020;146:2427-33.

11. Ren X, Cai X, Li J, et al. Histological transformation of lung adenocarcinoma to small cell lung cancer with mutant C797S conferring acquired resistance to osimertinib. J Int Med Res 2020;48:300060520927918.

12. Oser MG, Niederst MJ, Sequist LV, et al. Transformation from non-small-cell lung cancer to small-cell lung cancer: molecular drivers and cells of origin. Lancet Oncol 2015;16:e165-72.

13. Sequist LV, Waltman BA, Dias-Santagata D, et al. Genotypic and histological evolution of lung cancers acquiring resistance to EGFR inhibitors. Sci Transl Med 2011;3:75ra26.

14. Watanabe S, Sone T, Matsui T, et al. Transformation 
to small-cell lung cancer following treatment with EGFR tyrosine kinase inhibitors in a patient with lung adenocarcinoma. Lung Cancer 2013;82:370-2.

15. Offin M, Chan JM, Tenet M, et al. Concurrent RB1 and TP53 Alterations Define a Subset of EGFR-Mutant Lung Cancers at risk for Histologic Transformation and Inferior Clinical Outcomes. J Thorac Oncol 2019;14:1784-93.

16. Sementchenko VI, Watson DK. Ets target genes: past, present and future. Oncogene 2000;19:6533-48.

17. Yu HA, Arcila ME, Rekhtman N, et al. Analysis of tumor specimens at the time of acquired resistance to EGFR-TKI therapy in 155 patients with EGFR-mutant lung cancers. Clin Cancer Res 2013;19:2240-7.

18. Roca E, Gurizzan C, Amoroso V, et al. Outcome of patients with lung adenocarcinoma with transformation to small-cell lung cancer following tyrosine kinase inhibitors treatment: A systematic review and pooled analysis. Cancer Treat Rev 2017;59:117-22.

19. Ma S, He Z, Fu H, et al. Dynamic changes of acquired T790M mutation and small cell lung cancer transformation in a patient with EGFR-mutant adenocarcinoma after first- and third-generation EGFR-TKIs: a case report. Transl Lung Cancer Res 2020;9:139-43.

20. Basumallik N, Agarwal M. Small Cell Lung Cancer. StatPearls. Treasure Island, FL; 2020.

21. Lee JK, Lee J, Kim S, et al. Clonal History and Genetic Predictors of Transformation Into Small-Cell Carcinomas From Lung Adenocarcinomas. J Clin Oncol 2017;35:3065-74.

22. Forgacs E, Biesterveld EJ, Sekido Y, et al. Mutation

Cite this article as: Zhou Y, Bai H, Xia J, Xu WY, Cheng L, Xiong L. Novel ETV1 mutation in small cell lung cancer transformation resistant to EGFR tyrosine kinase inhibitors. Ann Transl Med 2021;9(14):1150. doi: 10.21037/ atm-21-2625 analysis of the PTEN/MMAC1 gene in lung cancer. Oncogene 1998;17:1557-65.

23. Peifer M, Fernández-Cuesta L, Sos ML, et al. Integrative genome analyses identify key somatic driver mutations of small-cell lung cancer. Nat Genet 2012;44:1104-10.

24. Johnson A, Severson E, Gay L, et al. Comprehensive Genomic Profiling of 282 Pediatric Low- and High-Grade Gliomas Reveals Genomic Drivers, Tumor Mutational Burden, and Hypermutation Signatures. Oncologist 2017;22:1478-90.

25. Matjasic A, Zupan A, Bostjancic E, et al. A novel PTPRZ1ETV1 fusion in gliomas. Brain Pathol 2020;30:226-34.

26. Lin MW, Su KY, Su TJ, et al. Clinicopathological and genomic comparisons between different histologic components in combined small cell lung cancer and nonsmall cell lung cancer. Lung Cancer 2018;125:282-90.

27. Liao S, Davoli T, Leng Y, et al. A genetic interaction analysis identifies cancer drivers that modify EGFR dependency. Genes Dev 2017;31:184-96.

28. Akazawa C, Sasai Y, Nakanishi S, et al. Molecular characterization of a rat negative regulator with a basic helix-loop-helix structure predominantly expressed in the developing nervous system. J Biol Chem 1992;267:2 1879-85.

29. Kovach C, Dixit R, Li S, et al. Neurog2 simultaneously activates and represses alternative gene expression programs in the developing neocortex. Cereb Cortex 2013;23:1884-900.

(English Language Editor: A. Kassem) 
Table S1 Genes list of the 639 DNA panel

\begin{tabular}{|c|c|c|c|c|c|c|c|c|c|c|c|c|}
\hline ABCB1 & BRD4 & COL5A1 & EPCAM & FGFR3 & HIST3H3 & KMT2B & MSI2 & PCNA & PTPRT & SETD8 & TEK & ZFHX3 \\
\hline АВСВ9 & BRIP1 & CREBBP & EPHA3 & FGFR4 & HLA-A & КМт2C & MST1 & PDCD1 & QKI & SF3B1 & TERT & ZNF217 \\
\hline ABL1 & BTG1 & CRKL & EPHA5 & $\mathrm{FH}$ & HLA-B & KMT2D & MST1R & PDCD1LG2 & RAB35 & SGK1 & TET1 & ZNF703 \\
\hline ABL2 & BTG2 & CRLF2 & EPHA7 & FLCN & HLA-C & KNSTRN & MTAP & PDGFRA & RAC1 & SH2B3 & TET2 & \\
\hline ACE2 & BTK & CSDE1 & EPHB1 & FLT1 & HLA-DRB1 & KRAS & MTHFR & PDGFRB & RAC2 & SH2D1A & TGFBR1 & \\
\hline ACVR1 & C10orf54 & CSF1R & EPHB4 & FLT3 & HMGB1 & LATS1 & MTOR & PDIA3 & RAD21 & $\mathrm{SHOC2}$ & TGFBR2 & \\
\hline ACVR1B & C11orf30 & CSF3R & EPHX1 & FLT4 & HMGN1 & LATS2 & MTRR & PDK1 & RAD50 & SHQ1 & TIPARP & \\
\hline AGO2 & C8orf34 & CTCF & ERAP1 & FOXA1 & HNF1A & LGALS9 & MUTYH & PDPK1 & RAD51 & SLC34A2 & TMEM127 & \\
\hline AKT1 & CALR & CTLA4 & ERAP2 & FOXL2 & HOXB13 & LGMN & MYB & PGR & RAD51B & SLCO1B1 & TMPRSS2 & \\
\hline AKT2 & CANX & CTNNA1 & ERBB2 & FOXO1 & HRAS & LIG1 & MYC & PHF6 & RAD51C & SLIT2 & TNF & \\
\hline AKT3 & CARD11 & CTNNB1 & ERBB3 & FOXP1 & HSD3B1 & LIG3 & MYCL & PHOX2B & RAD51D & SLX4 & TNFAIP3 & \\
\hline ALK & CARM1 & CTSB & ERBB4 & FRS2 & HSP90AA1 & LMO1 & MYCN & PIK3C2B & RAD52 & SMAD2 & TNFRSF14 & \\
\hline ALOX12B & CASP8 & CTSL & ERCC1 & FUBP1 & ICOSLG & LNPEP & MYD88 & PIK3C2G & RAD54L & SMAD3 & TNFRSF9 & \\
\hline AMER1 & CBFB & CTSS & ERCC2 & FYN & ID3 & LRP1B & MYOD1 & PIK3C3 & RAF1 & SMAD4 & TNFSF14 & \\
\hline ANKRD11 & $\mathrm{CBL}$ & CUL3 & ERCC3 & GABRA6 & IDE & LTK & NBN & PIK3CA & RANBP2 & SMARCA4 & TNFSF18 & \\
\hline APC & CBR3 & CUL4A & ERCC4 & GATA1 & IDH1 & LYN & NCOA3 & PIK3CB & RARA & SMARCB1 & TNFSF4 & \\
\hline AR & CCND1 & CXCR4 & ERCC5 & GATA2 & IDH2 & LZTR1 & NCOR1 & PIK3CD & RASA1 & SMARCD1 & TNFSF9 & \\
\hline ARAF & CCND2 & CYLD & ERF & GATA3 & $\mathrm{IFI} 30$ & MAF & NEGR1 & PIK3CG & RB1 & SMO & TOP1 & \\
\hline ARFRP1 & CCND3 & CYP17A1 & ERG & GATA4 & IFNGR1 & MAGI2 & NF1 & PIK3R1 & RBM10 & SMYD3 & TOP2A & \\
\hline ARID1A & CCNE1 & CYP19A1 & ERRFI1 & GATA6 & IGF1 & MALT1 & NF2 & PIK3R2 & RECQL & SNCAIP & TP53 & \\
\hline ARID1B & CD200 & CYP2C19 & ESR1 & GID4 & IGF1R & MAP2K1 & NFE2L2 & PIK3R3 & RECQL4 & socs1 & TP53BP1 & \\
\hline ARID2 & CD22 & CYP2C8 & ETV1 & GLI1 & IGF2 & MAP2K2 & NFKBIA & PIM1 & REL & SOD2 & TP63 & \\
\hline ARID5B & CD274 & CYP2C9 & ETV4 & GNA11 & IKBKE & MAP2K4 & NKX2-1 & PLCG2 & RET & sos1 & TP73 & \\
\hline ASXL1 & CD276 & CYP2D6 & ETV5 & GNA13 & $\mathrm{IKZF} 1$ & MAP3K1 & NKX3-1 & PLK2 & RFWD2 & SOX10 & TPMT & \\
\hline ASXL2 & CD40 & CYP3A4 & ETV6 & GNAQ & IL10 & MAP3K13 & $\mathrm{NOTCH} 1$ & PMAIP1 & RHEB & SOX17 & TPP2 & \\
\hline ATM & CD40LG & CYSLTR2 & EWSR1 & GNAS & IL7R & MAP3K14 & NOTCH2 & PMS1 & $\mathrm{RHOA}$ & sox2 & TRAF2 & \\
\hline ATR & CD48 & DAXX & EXO1 & GPR124 & INHA & MAPK1 & NOTCH3 & PMS2 & RICTOR & sox9 & TRAF7 & \\
\hline ATRX & CD70 & DCUN1D1 & $\mathrm{EZH} 1$ & GPS2 & INHBA & MAPK3 & NOTCH4 & PNRC1 & RIT1 & SPEN & TSC1 & \\
\hline AURKA & CD74 & DDR1 & $\mathrm{EZH} 2$ & GREM1 & INPP4A & MAPKAP1 & NPEPPS & POLB & RNF43 & SPOP & TSC2 & \\
\hline AURKB & CD79A & DDR2 & EZR & GRIN2A & INPP4B & MAX & NPM1 & POLD1 & ROS1 & SPRED1 & TSHR & \\
\hline AXIN1 & CD79B & DHFR & FAM175A & GRM3 & INPPL1 & MCL1 & NQO1 & POLE & RPS6KA4 & SPTA1 & TYMS & \\
\hline AXIN2 & CD80 & DICER1 & FAM46C & GSK3B & INSR & MDC1 & NRAS & PPARG & RPS6KB2 & SRC & TYRO3 & \\
\hline$A X L$ & CD86 & DIS3 & FAM58A & GSTP1 & IRF2 & MDM2 & NRD1 & PPM1D & RPTOR & SRSF2 & U2AF1 & \\
\hline B2M & CDA & DMD & FANCA & H3F3A & IRF4 & MDM4 & NSD1 & PPP2R1A & RRAGC & STAG2 & UGT1A1 & \\
\hline BABAM1 & CDC42 & DNAJB1 & FANCC & H3F3B & IRS1 & MED12 & NT5C2 & PPP2R2A & RRAS & STAT3 & UGT1A9 & \\
\hline BAP1 & CDC73 & DNMT1 & FANCD2 & H3F3C & IRS2 & MEF2B & NTHL1 & PPP4R2 & RRAS2 & STAT4 & UPF1 & \\
\hline BARD1 & $\mathrm{CDH} 1$ & DNMT3A & FANCE & HDAC1 & ITGAV & MEN1 & NTRK1 & PPP6C & RRM1 & STAT5A & VEGFA & \\
\hline ВBC3 & CDK12 & DNMT3B & FANCF & HERC1 & ITGB3 & MERTK & NTRK2 & PRDM1 & RSPO2 & STAT5B & VHL & \\
\hline BCL10 & CDK4 & DOT1L & FANCG & HGF & JAK1 & MET & NTRK3 & PRDM14 & RTEL1 & STK11 & VTCN1 & \\
\hline BCL2 & CDK6 & DPYD & FANCL & HIST1H1C & JAK2 & MGA & NUF2 & PREX2 & RUNX1 & STK19 & WHSC1 & \\
\hline BCL2L1 & CDK8 & DROSHA & FAS & HIST1H2BD & JAK3 & MICA & NUP93 & PRKAR1A & RUNX1T1 & STK40 & WHSC1L1 & \\
\hline BCL2L11 & CDKN1A & DUSP4 & FAT1 & HIST1H3A & JUN & МICB & NUTM1 & PRKCl & RXRA & SUFU & WISP3 & \\
\hline BCL2L2 & CDKN1B & DYNC2H1 & FBXW7 & HIST1H3B & KAT6A & MITF & P2RY8 & PRKD1 & RYBP & SUZ12 & WT1 & \\
\hline BCL6 & CDKN2A & E2F3 & FGF10 & HIST1H3C & KDM5A & MKNK1 & PAK1 & PRKDC & SDC4 & SYK & WWTR1 & \\
\hline BCOR & CDKN2B & EED & FGF12 & HIST1H3D & KDM5C & MLH1 & PAK3 & PRSS8 & SDHA & TAF1 & XIAP & \\
\hline BCORL1 & CDKN2C & EGFL7 & FGF14 & HIST1H3E & KDM6A & MLH3 & PAK7 & PTCH1 & SDHAF2 & TAP1 & $\mathrm{XPC}$ & \\
\hline BCR & CEBPA & EGFR & FGF19 & HIST1H3F & KDR & MPL & PALB2 & PTEN & SDHB & TAP2 & XPO1 & \\
\hline BIRC3 & CENPA & EIF1AX & FGF23 & HIST1H3G & KEAP1 & MRE11 & PARK2 & PTGS2 & SDHC & TAPBP & XRCC1 & \\
\hline BLM & CHD2 & EIF4A2 & FGF3 & HIST1H3H & KEL & MRE11A & PARP1 & PTP4A1 & SDHD & TAPBPL & XRCC2 & \\
\hline BMPR1A & CHD4 & EIF4E & FGF4 & HIST1H3I & KIT & $\mathrm{MSH} 2$ & PARP2 & PTPN11 & SESN1 & TBX3 & XRCC5 & \\
\hline BRAF & CHEK1 & ELF3 & FGF6 & HIST1H3J & KLF4 & $\mathrm{MSH} 3$ & PARP3 & PTPRD & SESN2 & TCEB1 & YAP1 & \\
\hline BRCA1 & CHEK2 & EP300 & FGFR1 & HIST2H3C & KLHL6 & MSH6 & PAX5 & PTPRO & SESN3 & TCF3 & YES1 & \\
\hline BRCA2 & $\mathrm{ClC}$ & EPAS1 & FGFR2 & HIST2H3D & KMT2A & MSI1 & PBRM1 & PTPRS & SETD2 & TCF7L2 & ZBTB2 & \\
\hline
\end{tabular}

The DNA panel is a hybridization capture-based NGS panel to detect single nucleotide variants (SNVs), insertion and deletion alterations (InDels), and copy number alterations (CNAs) involved in 639 cancer-associated genes in tumors. This panel is designed to provide mutation profiling of patients with solid tumors. 


\begin{tabular}{|c|c|}
\hline Primer & Sequences $\left(5^{\prime} \rightarrow 3^{\prime}\right)$ \\
\hline H-GAPDH-F & GGAGCGAGATCCCTCCAAAAT \\
\hline H-GAPDH-R & GGCTGTTGTCATACTTCTCATGG \\
\hline H-ETV1-F & TGGCAGTTITTGGTAGCTCTTC \\
\hline H-ETV1-R & CGGAGTGAACGGCTAAGTTTATC \\
\hline H-NOTCH1-F & TGGACCAGATTGGGGAGTTC \\
\hline H-NOTCH1-R & GCACACTCGTCTGTGTTGAC \\
\hline H-NOTCH2-F & ССTTCCACTGTGAGTGTCTGA \\
\hline H-NOTCH2-R & AGGTAGCATCATTCTGGCAGG \\
\hline H-ASCL1-F & CGCGGCCAACAAGAAGATG \\
\hline H-ASCL1-R & CGACGAGTAGGATGAGACCG \\
\hline H-HES1-F & ССTGTCATCCCCGTCTACAC \\
\hline H-HES1-R & CACATGGAGTCCGCCGTAA \\
\hline EGFR-WT-F & CTCGGATCCGCCACCATGCGACCCTCCGGGACGGC \\
\hline EGFR-WT-R & СССTCTAGACTCGAGTGCTCCAATAAATTCACTGCTT \\
\hline EGFR-L858R -F & CTCGGATCCGCCACCATGCGACCCTCCGGGACGGC \\
\hline EGFR-L858R -R & СССTCTAGACTCGAGTGCTCCAATAAATTCACTGCTT \\
\hline EGFR-T790M -F & CTCGGATCCGCCACCATGCGACCCTCCGGGACGGC \\
\hline EGFR-T790M -R & СССTCTAGACTCGAGTGCTCCAATAAATTCACTGCTT \\
\hline ETV1-WT-F & CTCGGATCCGCCACCATGGATGGATTTTATGACCAG \\
\hline ETV1-WT-R & СCCTCTAGACTCGAGATACACGTAGCCTTCGTTGTA \\
\hline ETV1-E462Q-F & CTCGGATCCGCCACCATGGATGGATTTTATGACCAG \\
\hline ETV1-E462Q-R & СCCTCTAGACTCGAGATACACGTAGCCTTCGTTGT \\
\hline ETV1-P159S-F & CTCGGATCCGCCACCATGGATGGATTTTATGACCAG \\
\hline ETV1-P159S-R & СССTCTAGACTCGAGATACACGTAGCCTTCGTTGT \\
\hline
\end{tabular}




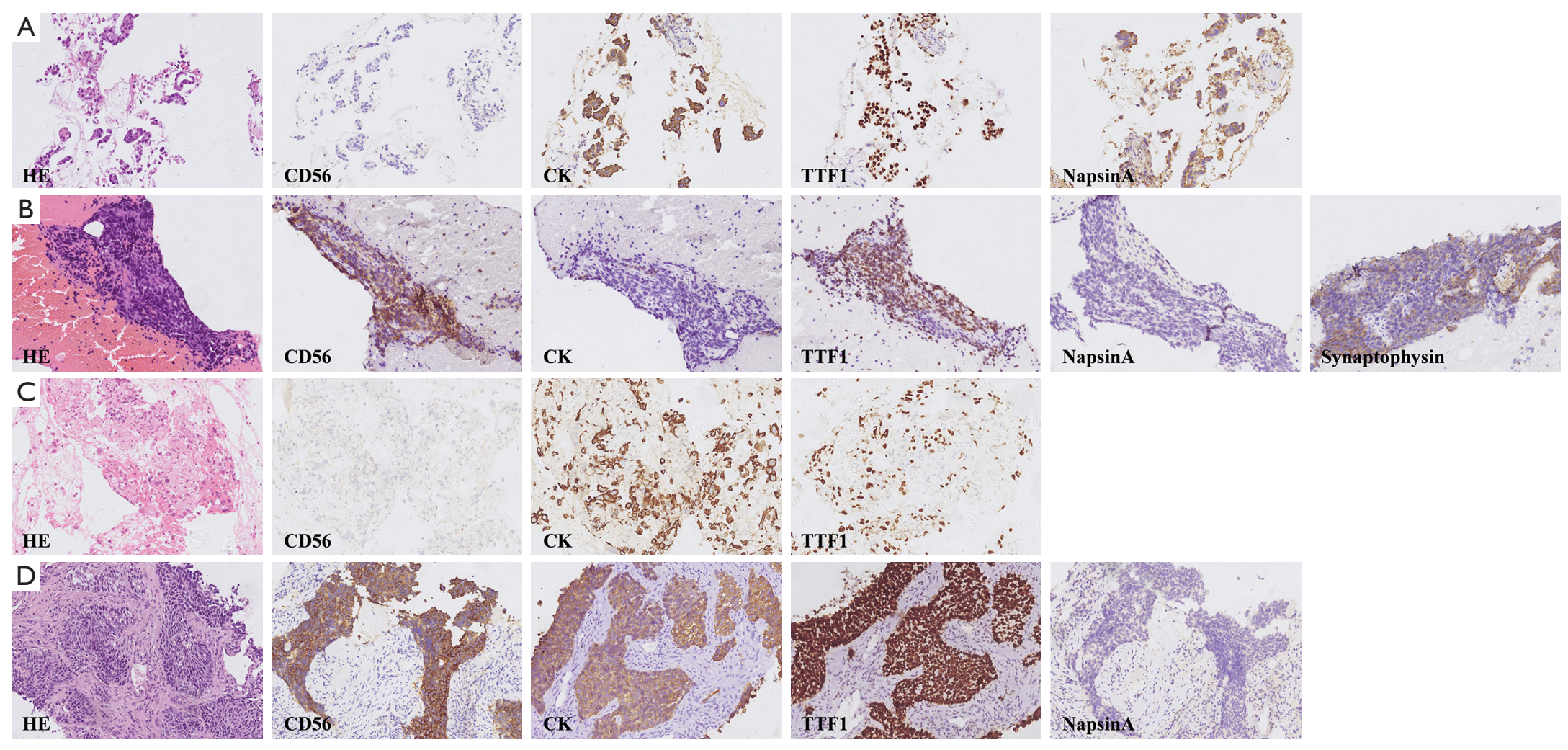

Figure S1 Pathological findings of the patients. (A) LUAD histology of case 1 before osimertinib treatment. IHC was strongly positive for CK, TTF1, and NapsinA, and negative for CD56 (200x). (B) Hematoxylin \& eosin staining showed that the cells were SCLC phenotype. IHC was positive for CD56 and synaptophysin, +/- for CK, partially positive for TTF1, and negative for NapsinA (200x). (C) LUAD histology of case 2 before EGFR-TKI treatment. IHC was strongly positive for CK and TTF1, and negative for CD56 (200x). (D) Re-biopsy after TKI resistance showed SCLC transformation. IHC was positive for CD56, CK, TTF1, and NapsinA (200x). 
A

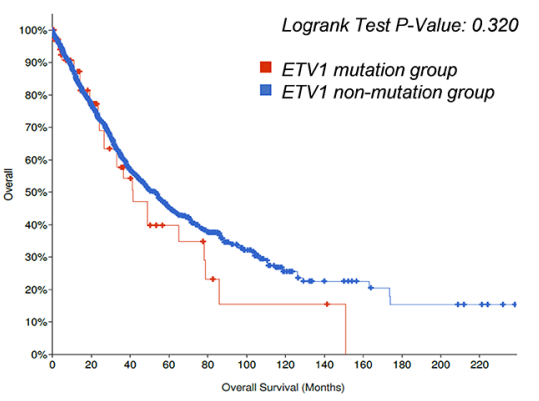

C

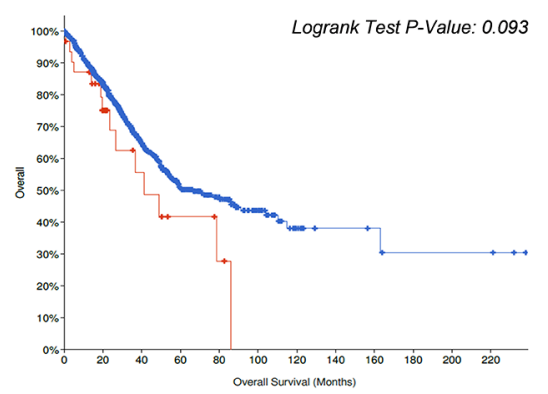

B

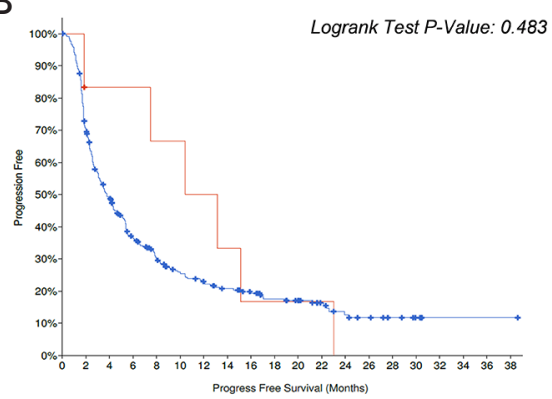

D

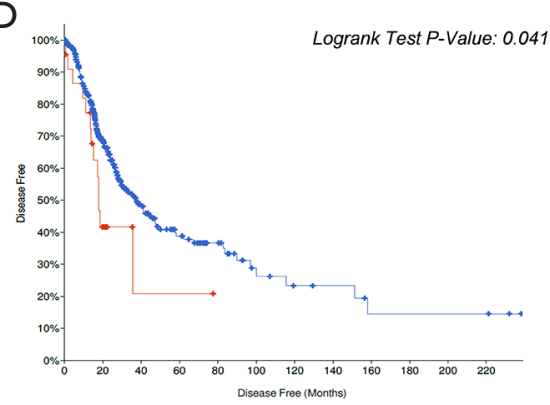

Figure S2 Survival association analysis of ETV1 mutations. (A) Kaplan-Meier OS analysis for tumor patients with and without ETV1 mutations in the TCGA cohorts. (B) PFS for tumor patients with and without ETV1 mutations. (C,D) OS and DFS analysis for LUAD patients with and without ETV1 mutations.

A

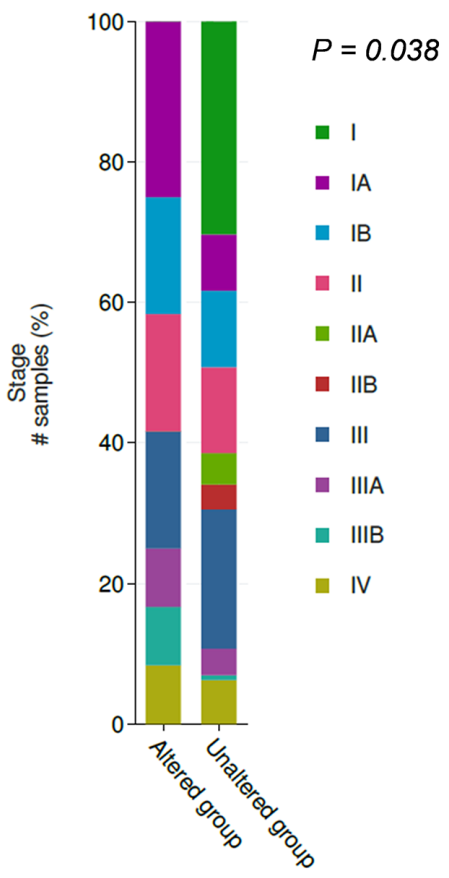

B

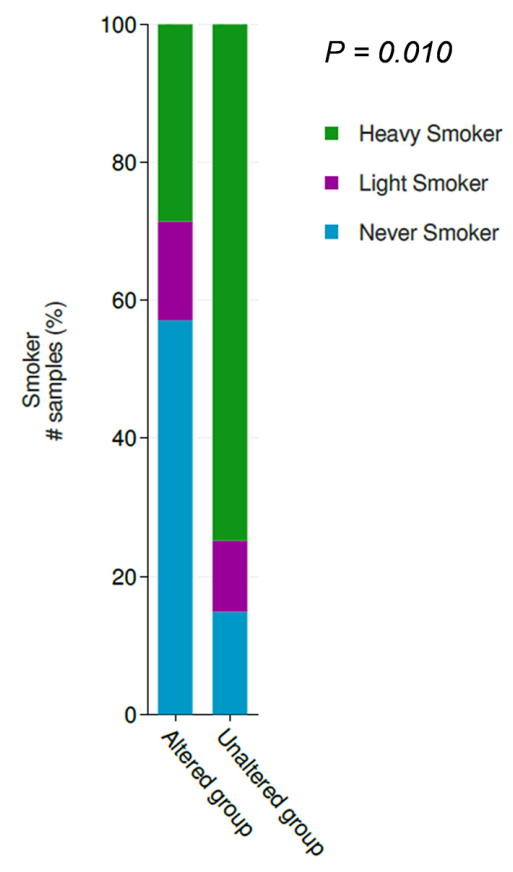

Figure S3 Clinical correlation analysis of ETV1 mutations. (A) Comparison of tumor stage between ETV1 mutated and non-mutated LUAD. (B) Smoking status and ETV1 mutations. 